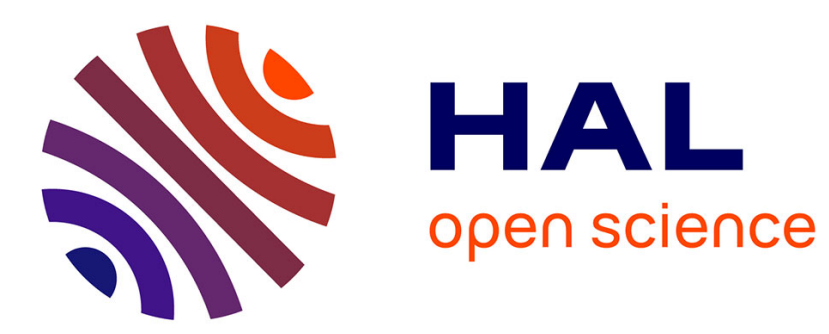

\title{
Construction royale et groupes culturels dans la Méditerranée médiévale: le cas de la Sicile à l'époque des souverains normands
}

Annick Peters-Custot

\section{- To cite this version:}

Annick Peters-Custot. Construction royale et groupes culturels dans la Méditerranée médiévale: le cas de la Sicile à l'époque des souverains normands. Le Moyen Age. Revue d'histoire et de philologie, 2012, CXVIII, pp.675-682. 10.3917/rma.183.0675 . halshs-03326323

\section{HAL Id: halshs-03326323 \\ https://shs.hal.science/halshs-03326323}

Submitted on 25 Aug 2021

HAL is a multi-disciplinary open access archive for the deposit and dissemination of scientific research documents, whether they are published or not. The documents may come from teaching and research institutions in France or abroad, or from public or private research centers.
L'archive ouverte pluridisciplinaire $\mathbf{H A L}$, est destinée au dépôt et à la diffusion de documents scientifiques de niveau recherche, publiés ou non, émanant des établissements d'enseignement et de recherche français ou étrangers, des laboratoires publics ou privés. 


\section{A. Peters-Custot.}

Construction royale et groupes culturels dans la Méditerranée médiévale : le cas de la Sicile à l'époque des souverains normands.

La publication d'un maillon important de la chaîne historiographique peut et doit être l'occasion de repréciser brièvement les positions antérieures et de présenter les acquis dus aux propositions nouvelles, ce qui est le propos de ce bref exposé, élaboré à partir du livre d'Annliese Nef, Conquérir et gouverner la Sicile islamique ${ }^{1}$. L'intérêt de cette récente publication nous parait en effet dépasser le seul champ de l'histoire de la Sicile médiévale, et mériter d'attirer l'attention des spécialistes d'autres espaces, d'autres périodes, voire d'autres domaines des sciences humaines et sociales. Les importantes mises au point que livre cette étude sur la construction d'un royaume médiéval, sur les groupes culturels méditerranéens, leur coexistence et leur participation à une telle création, sur les instruments de souveraineté et de domination, et leurs expressions de tout ordre, sont de nature à intéresser une communauté scientifique bien plus large que celle des seuls spécialistes de la Sicile médiévale.

L'ouvrage est issu d'une thèse dirigée par Henri Bresc et soutenue en décembre 2001. Le temps écoulé entre la soutenance et la publication a été celui d'une fertile maturation, qui a changé certaines analyses et conclusions, et jusqu'à la perspective même de ce travail, comme cela transparaît dans la modification du titre : la thèse portait en effet sur L'élément islamique dans la Sicile normande : identités culturelles et construction d'une nouvelle royauté $\left(\mathrm{XI}^{e}-\mathrm{XII}{ }^{e}\right.$ siècles). La disparition de l'expression d' «identités culturelles» est un des signes de l'évolution de la pensée de l'A. concernant les expressions de la culture collective, on y reviendra, tandis que le nouveau titre donne une vision plus dynamique et active, reflet d'une création royale et étatique, qui est au centre des thèses de l'A.. Ce livre paraît à l'École française de Rome qui a, de manière générale, pris une place éditoriale de premier plan dans le renouvellement des travaux historiques sur l'Italie méridionale et la Sicile médiévales, non seulement avec Henri Bresc ${ }^{2}$ et Jean-Marie Martin ${ }^{3}$ mais aussi, de manière très récente, avec des travaux de la génération suivante: Gian Luca Borghese ${ }^{4}$, Sulamith Brodbeck ${ }^{5}$, Philip Ditchfield $^{6}$, Benoît Grévin ${ }^{7}$, Annliese Nef bien entendu, Vivien Prigent (on espère vivement pouvoir disposer prochainement de la publication de sa thèse sur la Sicile byzantine ${ }^{8}$ ), ainsi que l'auteur de ces lignes ${ }^{9}$ et bien d'autres chercheurs encore.

\footnotetext{
${ }^{1}$ Annliese NEF, Conquérir et gouverner la Sicile islamique aux XI et XII siècles, Rome, 2011 (Bibliothèque des Écoles Françaises d'Athènes et de Rome [= désormais BEFAR], 346), 829 p.

${ }^{2}$ H. BRESC, Un monde méditerranéen. Économie et société en Sicile, 1300-1450, Rome, 1986 (BEFAR, 262).

3 J.-M. MARTin, La Pouille du vI e au XII siècle, Rome, 1993 (Collection de l'École française de Rome [= désormais CEFR], 179). On ajoutera également cette publication plus récente : Histoire et culture dans l'Italie byzantine, sous la dir. de A. Jacob, J.-M. Martin et G. Noyé, Rome, 2006 (CEFR 363).

${ }^{4}$ G.L. BorgheSE, Carlo I d'Angio e il Mediterraneo. Politica, diplomazia e commercio internazionale prima dei Vespri, Rome, 2009 (CEFR, 411).

${ }^{5}$ S. BRODBECK, Les saints de la cathédrale de Monreale en Sicile, Rome, 2010 (CEFR, 432).

${ }^{6} \mathrm{Ph}$. DITCHFIELD, La culture matérielle médiévale. L'Italie méridionale byzantine et normande, Rome, 2007 (CEFR, 373).

${ }^{7}$ B. GREVIN, Rhétorique du pouvoir médiéval. Les Lettres de Pierre de la Vigne et la formation du langage politique européen (XIII $-X V^{e}$ siècle), Rome, 2008 (BEFAR, 338).

${ }^{8}$ En attendant, notons la parution en cours des actes des cinq colloques qui se sont déroulés à Rome, avec la forte implication de l'École française de Rome, de 2008 à 2011 sur le thème de L'héritage byzantin en Italie, VIII ${ }^{e}$-XII $I^{e}$ siècles, dir. J.-M. MARTIN, A. PETERS-Custot et V. PRIGENT (ainsi que, pour le dernier colloque,
} 
Il est difficile de résumer ce qui paraît être, pour la Sicile des $\mathrm{XI}^{\mathrm{e}}$ et $\mathrm{XII}^{\mathrm{e}}$ siècles, une somme extraordinairement riche et destinée à constituer une référence par rapport à laquelle les spécialistes devront se positionner. L'intérêt de l'ouvrage déborde toutefois largement, comme on l'a dit, la seule histoire de la Sicile médiévale : ce livre embrasse et précise des notions fondamentales qu'il reprend, affronte, met à l'épreuve, et qui ont une valeur à la fois exemplaire et générale pour l'historien. Il donne surtout une vraie leçon de méthodologie historique en acte. Il ne s'agit pas uniquement de la maîtrise impressionnante et constante que l'A. montre de la bibliographie existant sur la Sicile médiévale, le dâr al-Islâm, le monde byzantin et l'Occident chrétien; de sa connaissance des enjeux historiographiques (marqués par l'ombre portée de Michele Amari ${ }^{10}$, et par les renouvellements récents dus notamment à Jeremy Johns ${ }^{11}$ ) ; et de la capacité de traitement de sources extrêmement hétérogènes. Cette maîtrise permet à l'A. de manifester une grande assurance et un ton affirmatif le plus souvent justifiés. Ce qui frappe, au-delà de ces qualités, est un mélange de ténacité et de rigueur scientifique, fait tout à la fois d'analyses scrupuleuses, de refus de la facilité, de maîtrise de l'écriture historique associant la capacité de synthétiser les recherches les plus précises à l'extrême prudence face à la généralisation. Toute proposition émise antérieurement est passée au même crible d'une nouvelle étude avant d'être acceptée (ou écartée), le silence est explicitement préféré à l'expression d'une éventuelle inexactitude dans l'état de la documentation. L'A. procède par dossiers, associant mise au point, discussion des théories précédentes et proposition d'une nouvelle interprétation, souvent originale dans sa méthode et sa démarche même (son étude du plafond de la Chapelle Palatine en est un bon exemple). La diversité des sources (chroniques, actes, inscriptions, monnaies, représentations sur des supports variés) impose une analyse chaque fois spécifique, qui prend en compte le contexte de réalisation, le mode de circulation, le public, l'accessibilité visuelle, etc. Quand une interprétation d'ensemble n'est pas possible dans le cadre actuel des connaissances, des « pistes » sont proposées.

Par ailleurs, l'A. affronte d'emblée la difficulté des mots et des notions d'usage courant, qui lui semblent à juste titre problématiques : la «tolérance » ou les «dominations » vues comme imposition unilatérale, par les vainqueurs, de leur culture aux vaincus; «l'influence ", concept vague et désincarné notamment en histoire de la culture et de l'art, la lecture «ethnique» des communautés, l'idée même "d'identité culturelle collective», difficilement appréciable à travers les sources médiévales, et qui implique une conception monolithique et stable de ce qui est en fait constamment en mouvement... Toute une terminologie qui, figeant les groupes dans des catégories fixes, évacue ce qui fait la complexité de l'histoire méditerranéenne et en particulier sicilienne: la fluidité qu'on rencontre en permanence, et qui n'empêche ni les conflits, ni la violence. L'A. ne se borne pas, du reste, à critiquer (au sens noble du terme), elle adapte et adopte (la distinction entre la sphère de la souveraineté et celle de la domination reprise de Michel Foucault ${ }^{12}$, structure la

Sulamith BRODBECK) : le premier volume est paru dans la Collection de l'École française de Rome : L'héritage byzantin en Italie (VIII ${ }^{e}$ XII $^{e}$ s.). La fabrique documentaire, éd. Rome, 2011 (CEFR, 449).

${ }^{9}$ A. Peters-Custot, Les Grecs de l'Italie méridionale post-byzantine. Une acculturation en douceur (IX ${ }^{e}-$ XIV ${ }^{e}$ s.), Rome, 2009 (CEFR, 420).

${ }^{10}$ M. AMARI, Storia dei Musulmani di Sicilia, rééd. et révision C. A. NALlino, Catane, 1933-1939, rééd. anast. Catane, 1991. Annliese Nef présente tout un bilan historiographique, notamment autour de Michele Amari dans Conquérir et gouverner la Sicile islamique, et qui pourra être complété par l'article suivant: A. NEF, Michele Amari ou l'histoire inventée de la Sicile islamique : réflexions sur la Storia dei Musulmani di Sicilia, MaghrebItalie : des passeurs médiévaux à l'orientalisme moderne (XIII -milieu XX $X^{e}$ siècle), éd. B. GREVIN, Rome, 2010 (CEFR, 439), p. 285-306.

${ }^{11}$ J. JoHNS, Arabic Administration in Norman Sicily. The Royal Dīwān, Cambridge, 2002.

${ }^{12}$ M. Foucault, "Il faut défendre la société ». Cours au Collège de France (1975-1976), dir. F. EWALD et A. FonTANA, Paris, 1997. 
réflexion et même une bonne partie de l'ouvrage, en permettant de lier constamment histoire politique et histoire sociale), et elle propose également, dans des développements extrêmement stimulants : que ce soit l'idée des «rythmes» de motifs iconographiques du plafond de la Chapelle palatine, qui créent à la fois la stupeur et la familiarité chez l'observateur trop éloigné pour discerner les subtilités iconographiques ; ou encore l'idée d'un consensus nécessaire de la population à la souveraineté, y compris dans le cadre monarchique médiéval, et qui apparaît encore plus clairement dans la Sicile de l'époque normande, où une minorité victorieuse ne saurait dominer durablement une population majoritaire en n'employant que la contrainte et la violence ${ }^{13}$; ou enfin, pour remplacer la vaporeuse «influence », la notion d'emprunt conscient. Pour ce qui ne trouve pas d'expression rigoureusement exacte, l'A. propose de se contenter de propositions, en ayant conscience de leurs limites (les «groupes culturels » par exemple).

Le but assigné à cet ample travail est de cerner « les modalités de domination mises en place par les Hauteville et ceux qui les entouraient en Sicile, dans un contexte où la majorité de la population était arabo-musulmane » (p. 14) avec comme défi principal la place de l'islâm et des musulmans dans une construction élaborée sur la base d'héritages islamiques (et, en moindre mesure, byzantins). Pour atteindre ce but, l'A. analyse la nature d'un pouvoir non seulement à travers son idéologie, mais aussi sa mise en place, la manifestation de l'État et de l'administration, la nature et le statut des élites, les partitions territoriales, et la gestion des populations. Si la construction claire et progressive de l'ensemble présente une grande cohérence, il n'en reste pas moins que ces 829 pages constituent de fait deux livres en un. Les deux premières parties, unissant la souveraineté et la domination dans ses multiples aspects ( «les langues du roi », la conception du pouvoir et sa mise en scène - titulatures, images du roi, processions, Chapelle palatine, mécénat architectural et littéraire - l'État, l'administration, le service du souverain et le milieu curial) décrivent et décortiquent au prisme d'une documentation trilingue d'une grande diversité générique, le pouvoir des souverains normands de Sicile dans ses aspects idéologiques et dans ses réalisations pratiques, attestant la créativité mise en œuvre dans leur construction d'un État, pour laquelle ils adaptent des éléments islamiques (mais pas seulement) et récupèrent à leur service les élites arabo-musulmanes (entre autres). Les deux parties suivantes observent pour l'essentiel, à partir d'une documentation vaste, mais moins diversifiée (essentiellement les actes notariés et les listes d'hommes en arabe ou en arabe et grec, les jarâ'id) les structures territoriales (organisation du territoire, composition des élites, féodalisme et féodalité, statuts juridiques des hommes, stratification et fluidité sociale). On y trouvera en particulier une recherche décisive sur les statuts des hommes recensés dans les jarâ'id, et une interprétation stimulante de la jiziya inversée, impôt de capitation et symbole de soumission que les Normands ont fait peser sur les populations arabo-musulmanes (et les juifs) comme il pesait sur les dhimmî dans la Sicile islamique.

Ces deux grands ensembles sont encadrés de manière originale par deux parties à première vue plus événementielles, mais dans lesquelles les faits sont présentés dans la perspective de questionnements de fond: un préambule sur la conquête de l'île par les Normands met en place les éléments du long débat sur l'identification ou non de cette entreprise à une croisade ; un épilogue, fondé sur l'étude des chroniques qui révèlent les zones conflictuelles souvent masquées dans les sources utilisées précédemment, met en perspective

\footnotetext{
${ }^{13}$ On peut ainsi rapprocher la pensée de l'A. sur la construction royale siculo-normande des réflexions de Pierre Bourdieu sur l'État, et en particulier sur la nécessité, pour l'État et même dans les sociétés sans État, d'une organisation du consentement commun, notion à la quelle l'A. ajoute, de son côté, l'importance de l'imaginaire politique (P. Bourdieu, Sur l'État. Cours au Collège de France [1989-1992], Paris, 2012). La primauté du politique, qui implique décision, arbitrage, création, permet de lier entre elles des catégories le plus souvent artificiellement distinguées (le politique, le social, le religieux).
} 
les évolutions de la politique intérieure des souverains Hauteville d'un côté, et les balancements de leur politique méditerranéenne, en particulier face aux États du dâr al-Islâm, de l'autre.

La conclusion générale, dense et affirmative à l'image de tout le livre, tout en soulignant une fois encore les éléments qu'on ne percevra jamais en raison des lacunes documentaires, reprend les interprétations qu'on peut raisonnablement admettre et celles que l'A. estime nécessaire d'abandonner. Soucieux de se défaire simultanément de la vision de l'État normand de Sicile comme d'un État «moderne», et comme d'un État fictif et inefficace, l'A. souligne la créativité administrative et idéologique qui est mise en œuvre de manière évolutive sous la forme d'un «bricolage» pragmatique, et qui sous-tend une construction politique et administrative centralisée à Palerme, suffisamment efficace pour financer les politiques ambitieuses des Hauteville sans écraser la population (sauf à la fin de la période, ce qui n'est qu'une des causes de la dégradation des conditions de vie des campagnes). Le critère culturel est sérieusement nuancé au sens où, si la présence arabomusulmane est puissante à la cour (ce que reflète le rôle des eunuques), et si le langage politique et administratif est en partie repris de ce que les Normands trouvèrent en arrivant en Sicile, l'altérité culturelle des sujets siciliens des souverains n'est jamais envisagée de manière radicale, et à l'inverse l'acceptation ou le refus de la construction normande n'est pas déterminée, dans la population, sur des bases culturelles, ne serait-ce que parce que les groupes ne sont en aucun cas clos sur eux-mêmes, ni dans les faits, ni dans la perception des contemporains. La fin de la période voit toutefois la remise en cause de cette création qui, le fait est à souligner, ne servit pas de modèle. La dégradation sensible des conditions économiques d'une large part de la population est un facteur explicatif non négligeable de l'ébranlement de cette construction. Paradoxalement, les choix de l'arabisation et de l'islamisation d'une partie de l'administration et des rituels politiques ont durci les oppositions entre groupes, pourtant délimités de manière souple. Or politique intérieure et politique méditerranéenne sont en relation étroite, si bien que, lorsque l'horizon africain s'éloigne, après Roger II et Guillaume $\mathrm{I}^{\mathrm{er}}$, au profit d'un attachement de plus en plus continental, l'arabisation se justifie moins, et l'opposition entre les grands latins et les administrateurs arabo-musulmans se fait plus sensible, au prix de secousses politiques rarement pacifiques.

Faute de pouvoir rendre compte, dans le format d'un court article, de l'ensemble des sujets abordés et des interprétations proposées, on s'arrêtera ici, de manière un peu arbitraire, car déterminée par les intérêts propres de l'auteur de ces lignes, sur la conception de la royauté normande de Sicile, considérée par le biais des « images du roi » et du mécénat royal et de cour. La grande question qui unit ces développements est celle des emprunts à l'héritage islamique de la Sicile, dans la formulation d'une idéologie siculo-normande de la royauté. Dans ce domaine, centrale est la question des transferts culturels au Moyen Âge. Leurs origines, leurs évolutions, leurs réinterprétations sont rarement identifiables avec certitude, en raison des points communs nombreux entre Empire byzantin et traditions fatimides dans les expressions du pouvoir, artistiques ou non : ces ensembles puisent aux mêmes sources (les Empires romain et sassanide) qui ont permis d'élaborer "une koinè de la souveraineté » (p. 143). Par ailleurs, les éléments repris sont toujours réadaptés. Toujours est-il que l'A. conclut sur l'inventivité des Hauteville, qui s'appuient largement sur des éléments islamiques pour manifester une conception sacrée de leur royauté, mais dans le cadre d'un christianisme arabisé qui devient le berceau de l'unité du royaume, et dont l'universalité rejaillit sur la gloire du roi, elle-même à vocation universelle.

Si les analyses sont pleinement convaincantes voire novatrices, les conclusions qui en découlent reposent également sur les limites que 1'A. a explicitement données à son domaine 
d'étude : l'élément islamique sicilien. Ainsi, en revendiquant de ne travailler, pour son étude des «images du roi », que sur "les éléments couramment considérés comme "islamiques" » (p. 119), l'A. expulse volontairement quantité de modes de représentations du souverain. Or les rois normands gouvernent la Sicile islamique, mais pas seulement, et il est fort probable que l'idéologie royale qu'ils mettent en place, non seulement s'exprime de manière différenciée et parfois non islamique dans la Sicile même, mais doit prendre en compte le fait qu'une même royauté domine ailleurs qu'en Sicile. Si la Sicile islamique, et Palerme en particulier, sont au cœur de cette construction, celle-ci doit être adaptable au contexte péninsulaire et à tous les sujets siciliens et italiens du royaume, faute de pouvoir créer le consensus nécessaire. Le roi doit être reconnu de tous.

Signalons d'abord qu'un ordo de couronnement royal remontant probablement à Roger II et étudié par Reinhard Elze ${ }^{14}$ est attesté dans quatre manuscrits issus du royaume de Sicile. Cet ordo s'inspire du pontifical romano-germanique du $\mathrm{X}^{\mathrm{e}}$ siècle : de ce point de vue, le roi de Sicile est un souverain occidental. En revanche, dans les églises siciliennes «royales », ou proches du roi (Martorana, Monreale), le roi normand est représenté en basileus. On sait que ce sont des représentations qui n'ont rien à voir avec la réalité, ni des habits et costumes du roi, ni des habits du basileus au XII ${ }^{\mathrm{e}}$ siècle (particulièrement pour la mosaïque de la Martorana, copiée sur un modèle iconographique byzantin vieux de deux siècles ${ }^{15}$ ). Toutefois, le choix de la représentation publique du roi en basileus et non à la mode occidentale ne peut être considéré comme anodin. Ce choix a forcément un sens : tel le basileus, le roi Roger de la Martorana, Christostéphanitzos (couronné par le Christ), ne tient son pouvoir d'aucune autorité terrestre. Si l'expression arabe du christianisme est un élément incontestable de la construction idéologique siculo-normande, elle n'est pas la seule, et l'élection impériale byzantine contribue aussi, une fois adaptée par les Hauteville, à manifester une dimension sacrale du roi. Certes, jamais les rois normands ne se parent du titre de basileus, réservé à l'empereur byzantin : l'usage du terme de rex translittéré en lettres grecques ( $\dot{\rho} \grave{\eta} \xi)$ ressortit à la même prudence que celui de malik en arabe - outre qu'il est le titre concédé par les empereurs byzantins aux rois occidentaux ${ }^{16}$. Toutefois, les répliques normandes de l'idéologie impériale byzantine sont indéniables, et elles ne se limitent pas à l'iconographie.

Dans le domaine de l'épigraphie monumentale, l'inscription trilingue très fameuse de l'horloge hydraulique de Palerme donne lieu à une interprétation des trois textes arabe, grec, latin par l'A. (p. 184-185) : un texte latin sobre, un texte grec qui définit l'horloge comme clepsydre, et un texte arabe qui, muni d'une titulature royale très ample, renvoie, d'après l'A. «à la figure d'un souverain savant et dévoué à la protection de son royaume », contrôleur des heures - comme de son administration. Toutefois le texte grec, ici translittéré en caractères latins (la translittération de textes grecs d'une certaine longueur montre d'ailleurs ici ses limites, notamment pour les nombres) est accompagné d'une traduction qui ne le rend pas

\footnotetext{
${ }^{14}$ R. ElzE, Zum Königtum Rogers II. von Sizilien, Festschrift Percy Ernst Schramm zu seinem siebzigsten Geburtstag, I, Wiesbaden, 1964, p. 102-116.

${ }^{15}$ Le couronnement de Roger II par le Christ dans l'église de S. Maria dell'Ammiraglio, dite aussi Martorana, est en effet la copie conforme d'un ivoire byzantin représentant la même scène, avec un basileus du nom de Constantin, et daté du milieu du $\mathrm{X}^{\mathrm{e}}$ siècle. Cet empereur ne peut être que Constantin VII Porphyrogénète (dont le règne personnel commence en janvier 945). Cet ivoire, trouvé dans le Sud du Caucase, se trouve actuellement au musée Pouchkine de Moscou. Il est possible toutefois que plusieurs exemplaires similaires aient été sculptés à l'occasion du couronnement de Constantin VII Porphyrogénète, et que l'un d'entre eux se soit retrouvé dans l'Italie méridionale byzantine, ou y ait été apporté ultérieurement. On peut se référer à A. CUTLER, The Hand of the Master. Craftmanship, Ivory, and Society in Byzantium (9th-11th Centuries), Princeton, 1994, en particulier p. 203-235 et à E. KitZINGER, The mosaics of St Mary's of the Admiral in Palermo, Washington, 1990 (Dumbarton Oaks Studies, 27).

${ }^{16}$ J.-M. MARTIN, L'Occident chrétien dans le Livre des Cérémonies, II, 48, Travaux et Mémoires du Centre d'Histoire et de Civilisation de Byzance, 13, 2000, p. 617-646.
} 


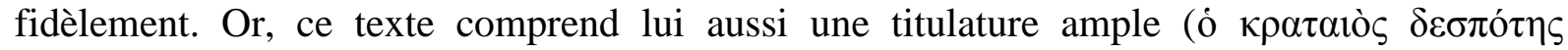

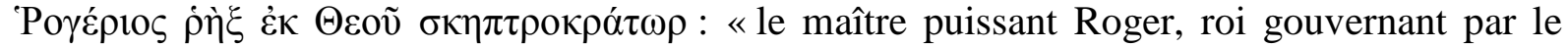
sceptre tenu de Dieu»), et montre le souverain diriger le cours de l'eau et produire une connaissance infaillible des heures («[le roi] dirige le cours de l'élément qui coule, en accordant une connaissance infaillible des heures de l'année ${ }^{17}$ ) ce qui est présenté comme un nouveau miracle (' $\Omega \theta \alpha \tilde{v} \mu \alpha$ Kaıvóv). C'est une version finalement assez proche de la version arabe, et qui magnifie le souverain dans des termes similaires et tout aussi solennels.

De même, après son étude de la production littéraire à la cour des Hauteville et sous le mécénat des souverains, l'A. conclut (p. 202) que «le domaine le plus fréquenté par les auteurs de langue grecque proches de la cour est donc la théologie ». On ne saurait être d'accord avec cette interprétation, qui classe dans la théologie la description de la Chapelle palatine par Philagathe de Cerami, le panégyrique de Guillaume $\mathrm{I}^{\mathrm{er}}$ par Eugène de Palerme, et surtout la Taxis des trônes patriarcaux de Nil Doxopater, Byzantin exilé à la cour de Roger II. Le traité de Nil, qui défend la soumission historique des sièges de l'ex-Italie méridionale byzantine et de la Sicile au patriarcat de Constantinople, sert les prétentions ecclésiastiques des souverains normands aussi bien que le parallèle iconographique avec le basileus; il a donc valeur ecclésiologique. Les éléments byzantins dans la littérature curiale et l'art palermitain se focalisent donc sur des thèmes précis, qui ne sont pas forcément de l'ordre du « religieux » (adjectif trop vague), mais de l'indépendance par rapport à la papauté ; ils sont donc investis d'un message politique, magnifiant un roi chef de son Église (alors même que le « contrat » passé à Melfi entre Robert Guiscard et le pape en 1059 aurait dû faire du souverain normand un réel vassal du pape) sur laquelle son pouvoir est pratiquement sans limite (au moment même de la réforme pontificale et de l'exigence de libertas Ecclesie).

La question de l'héritage ou de l'importation d'éléments byzantins en Sicile aurait donc pu être évoquée plus profondément : l'arrivée de personnes de culture byzantine à la cour de Roger II n'est pas absente de ce travail ; l'héritage manque un peu, issu de la transmission, soit de traces byzantines qui auraient pu perdurer dans la Sicile islamique, soit d'éléments issus de la Calabre méridionale, dont on connaît le degré de byzantinisation au moment de l'arrivée des Normands, et que ces derniers ont fini de pacifier juste avant de se lancer à la conquête de l'île.

De ce fait, on ne peut que souligner une lacune concernant ce qui devrait être vu comme une annexe péninsulaire de la Sicile, et en particulier du Val Demone : la Calabre méridionale. Par moments, l'A. évoque à titre comparatif les Abruzzes (étudiées par Laurent Feller ${ }^{18}$ ) ou la Pouille (objet principal des recherches de Jean-Marie Martin ${ }^{19}$ ). En revanche, la Calabre méridionale est reléguée dans un quasi-silence, alors même que de nombreux travaux ont été réalisés sur son histoire politique, administrative, culturelle, l'héritage byzantin, les statuts paysans, ou la politique ecclésiastique des Hauteville ${ }^{20}$, mais qui, même cités, sont

\footnotetext{
${ }^{17}$ La traduction de ce bref passage est celle que propose André Guillou dans Recueil des inscriptions grecques d'Italie médiévale, Rome, 1996 (CEFR, 222), n 198 p. 216-218, ici p. 216.

${ }^{18}$ L. FELLER, Les Abruzzes médiévales : territoire, économie et société en Italie centrale du IX au XII siècles, Rome, 1998 (BEFAR, 300).

${ }^{19}$ Notamment J.-M. MARTIN, La Pouille, cité supra note 3.

${ }^{20}$ On se permet de citer: A. PETERS-CUSTOT, Les Grecs de l'Italie méridionale (supra note 8); EAD., Les remaniements de la carte diocésaine de l'Italie grecque lors de la conquête normande: une politique de latinisation forcée de l'espace? (1059-1130), Pouvoir et territoire, Colloque du CERHI, Saint-Etienne, 78 novembre 2005, éd. P. RODRIGUEZ, Saint-Etienne, 2007 (Travaux du CERHI, 6), p. 57-77 ; EAD., Gli elenchi di uomini, Studi in margine all'edizione della Platea di Luca arcivescovo di Cosenza (1203-1227), E. CUOZZO et J.-M. MARTIN éd., Avellino, 2009, p. 141-158; dans le même volume, S. CAROCCI, Angararii e franci. Il villanaggio meridionale, p. 205-241 (et, plus généralement, toutes les contributions de ce volume) ; A. PETERSCustot, Brébion, kodex et plateae : petite enquête sur les instruments de la propriété monastique dans la Calabre méridionale aux époques byzantine et normande, Puer Apuliae. Mélanges en l'honneur de Jean-Marie Martin, dir. E. Cuozzo, V. Deroche, A. Peters-Custot et V. Prigent, 2 volumes, Paris, 2008 (Centre de
} 
rarement exploités par l'A. de manière à fournir une autre vision, plus locale, moins décisive, mais également intéressante, de la royauté des Hauteville. Si la Calabre méridionale n'abrite plus la capitale au début du XII ${ }^{\mathrm{e}}$ siècle, elle faisait partie du même ensemble administratif que la Sicile et connaît aussi ces listes nominales de paysans dressées par l'autorité publique (katonoma), équivalents grecs des jarâ'id, sans avoir forcément le même sens ; la chancellerie des Hauteville, trilingue, écrivait aussi pour la Calabre méridionale et en grec, même pour des monastères latins ; le comte, puis le roi, est également maître de la politique ecclésiastique de la région, pourtant structurellement différente de la Sicile par sa population chrétienne, de langue grecque, de droit byzantin, par sa grande propriété d'époque byzantine et aussi par son enclavement.

D'autres perspectives de recherche s'annoncent déjà dans ce travail riche en érudition comme en réflexion, et l'A. a de fait déjà suggéré, dans une imposante contribution prospective réalisée avec Vivien Prigent, les pistes de recherche que devrait suivre le profond renouvellement souhaitable de l'histoire de la Sicile médiévale ${ }^{21}$. En particulier, l'A. regrette, dans son ouvrage, que la maigre connaissance dont on dispose actuellement sur la Sicile islamique ne permette pas d'interpréter dans toutes ses dimensions la construction normande. Certains indices concernant de probables continuités byzantines pendant l'époque islamique montrent que cette autre période de transition, entre une Sicile byzantine de moins en moins méconnue grâce aux recherches fondées sur l'archéologie, la sigillographie et la numismatique, et la Sicile islamique, mérite d'être reconsidérée, malgré les lacunes documentaires, avec la même rigueur que celle dont l'A. a fait preuve pour la Sicile normande. On sait que l'A. travaille en ce sens, et on s'en félicite, à la vue des qualités exceptionnelles de son travail sur la Sicile à l'époque normande.

recherche d'histoire et de civilisation de Byzance. Monographies, 30), II, p. 537-552. Certaines contributions des cinq colloques du programme L'héritage byzantin en Italie, VIII - -XII siècles (cf. supra note 8 ) concernent l'Italie méridionale, Pouille et Calabre en particulier. Annliese Nef a participé à la quatrième rencontre, portant sur les structures rurales, les 17 et 18 décembre 2010.

${ }^{21}$ A. NEF et V. PRIGENT, Per una nuova storia del Medioevo siciliano, Storica, 33, 2006, p. 9-63. 